\title{
O modelo transteorético aplicado ao consumo de frutas e hortaliças em adolescentes
}

\author{
The transtheoretical model applied for \\ fruit and vegetable consumption among \\ adolescents
}

Maria do Carmo Fontes de OLIVEIRA ${ }^{1}$

Gisele Kuhlmann DUARTE²

\section{RES U M O}

\section{Objetivo}

Identificar a relação entre dois constructos do Modelo Transteorético - estágios e processos de mudança - quanto ao consumo de frutas e de hortaliças por adolescentes.

\section{Métodos}

Em desenho transversal, foi aplicado um questionário a 61 adolescentes, verificando que o uso do processo autoliberação se distinguiu significativamente $(p<0,05)$ em todos os estágios de mudança no consumo de frutas e de hortaliças.

\section{Resultado}

O processo suporte social apresentou diferenças significativas $(p<0,05)$ no seu uso entre estágios pré-contemplação/contemplação e preparação apenas no consumo de frutas. Quanto ao uso do processo auto-reavaliação, foram encontradas diferenças significativas $(p<0,05)$ entre os estágios pré-contemplação/ contemplação e preparação no consumo de hortaliças.

\section{Conclusão}

Concluiu-se que alguns processos específicos de mudança eram utilizados pelos adolescentes ao se moverem por meio dos estágios de mudança. Intervenções empregando tais processos devem ser realizadas para possibilitar avanços nos estágios de mudança do consumo de frutas e de hortaliças.

Termos de Indexação: comportamento do adolescente; frutas; hortaliças.

\footnotetext{
1 Departamento de Nutrição e Saúde, Centro de Ciências, Universidade Federal de Viçosa. Avenida P.H. Rolfs, s/n, 36570-000, Campus Universitário, Viçosa, MG, Brasil. Correspondência para/Correspondence to: M.C.F. OLIVEIRA. E-mail: $<$ mcfonoli@ufv.br>.

2 Bolsista PIBIC/CNPq, Curso de Nutrição, Universidade Federal de Viçosa, Viçosa, MG, Brasil.
} 


\section{A B S T R A C T}

\section{Objective}

The purpose of this study was to identify the relationship between two constructs of the Transtheoretical Model - stages and processes of change - related to the consumption of fruits and vegetables by adolescents.

\section{Methods}

In a cross-sectional study, 61 adolescents were surveyed. The self-liberation process was significantly distinguished $(p<0.05)$ in all stages of change in the consumption of fruits and vegetables.

\section{Results}

The social support process presented significant differences $(p<0.05)$ between the precontemplation/ contemplation and preparation stages of change only in fruit consumption.

\section{Conlusion}

With respect to the use of the self-reassessment process, significant differences were found $(p<0.05)$ between precontemplation/contemplation and preparation stages in vegetable consumption. Some specific processes of change were used by adolescents when moving through the stages of change. Interventions using these processes should be done to allow advances in the stages of change in the consumption of fruits and vegetables.

Indexing terms: adolescent behavior; fruits; vegetables.

\section{N T R O D U Ç Ã O}

Entender o processo da mudança dietética da população em geral é importante para o desenvolvimento de efetivas intervenções em nutrição ${ }^{1,2}$. O Modelo Transteorético proposto por Prochaska et al. ${ }^{3}$ tem potencial para explicar e para facilitar mudanças em uma variedade de comportamentos relacionados à saúde $\mathrm{e}^{4-9}$.

O modelo sugere que, embora as pessoas percebam que precisam realizar mudanças em seus comportamentos, elas as fazem em estágios ao invés de realizarem uma mudança considerável e abrupta. Durante esses estágios, a pessoa pensa sobre o problema de comportamento, considera o que fazer e decide quando entrar ou não em ação ${ }^{10}$. Os estágios de mudança representam a dimensão temporal que permite segmentar e entender quando ocorrem mudanças na intenção, na atitude e no comportamento nutricional. No modelo, cada estágio de mudança representa não só um período de tempo, como também um conjunto de tarefas necessárias à movimentação para o próximo estágio ${ }^{3}$.

Há cinco estágios de mudança que podem ser descritos em determinada seqüência. O primeiro estágio, pré-contemplação, não apresenta a intenção de modificar o comportamento, e os indivíduos não têm consciência de seus comportamentos inadequados. O segundo estágio, chamado de contemplação, é aquele em que ocorre a identificação do problema de comportamento, e as pessoas começam a considerar uma possibilidade de mudança, sem terem iniciado a agir nesse sentido. Trata-se de um período de ambivalências, em que são avaliados as barreiras e os benefícios para a mudança. Já o terceiro estágio, o da preparação, é aquele em que são desenvolvidos os planos específicos de ação, e são experimentadas pequenas mudanças de comportamento. O quarto estágio é o da ação, momento em que os planos de mudança são concretizados. Por fim, o último estágio, o da manutenção, é quando as pessoas trabalham para consolidarem a mudança, prevenindo recaídas. As pessoas só estarão no estágio de manutenção quando alterarem o comportamento por um período de tempo maior do que seis meses.

Os processos de mudança compreendem a segunda dimensão do modelo e envolvem estratégias, técnicas e intervenções que 
possibilitam aos indivíduos mudarem de comportamento com sucesso, movendo-se por meio dos cinco estágios de mudança. Esses processos, além de permitirem a compreensão de como as intenções de mudança ocorrem, podem ser divididos em duas categorias: processos cognitivos e processos comportamentais.

Os processos cognitivos são cinco e envolvem mudanças nas atitudes dos indivíduos: liberação social - processo que envolve o aumento da disponibilidade de comportamentos alternativos na sociedade para que o indivíduo possa mudar seu comportamento; aumento de consciência - elevação do nível de consciência do indivíduo sobre si mesmo e sobre o comportamento não desejável; auto-reavaliação - avaliação de si próprio quanto aos custos e benefícios da mudança de comportamento; reavaliação ambiental - julgamento de como o próprio problema de comportamento afeta o ambiente pessoal e físico; e alívio dramático - etapa que envolve experimentar e expressar sentimentos sobre si próprio em relação ao seu problema de comportamento.

Os processos comportamentais são definidos pelas atividades que os indivíduos executam para modificarem o comportamento problemático e podem ser, assim, definidos: contracondicionamento, baseado na substituição do comportamento não desejável por um comportamento alternativo; autoliberação, relacionada a mudanças em nível experiencial que aumentam a capacidade de o indivíduo fazer escolhas, realizar atos e acreditar na possibilidade de mudança; controle de estímulos, ligado a evitar ou contornar estímulos que levam ao comportamento indesejável; administração de contingências, baseado em enfatizar e premiar a mudança de comportamento; e suporte social, relacionado ao fato de os indivíduos estarem abertos e dispostos a confiarem em pessoas que se importam com eles.

O elemento-chave do Modelo Transteorético é a relação entre os estágios e os processos de mudança. Os processos são diferentemente usados no decorrer dos estágios, pois, durante a fase de pré-contemplação, os indivíduos usam os processos significativamente menos que pessoas em outros estágios. Porém, indivíduos no estágio de contemplação são mais abertos aos processos cognitivos, tais como: técnicas de aumento de consciência, alívio dramático e reavaliação do ambiente.

O constructo estágio de mudança do Modelo Transteorético tem sido investigado no contexto de numerosos comportamentos relacionados à saúde. Para ilustrar, podem-se mencionar os estudos de redução de gordura de Brug et al. ${ }^{11}$, Steptoe et al. ${ }^{12}$, Sporny et al. ${ }^{13}$ e Rossi et al. ${ }^{14}$, e aqueles estudos sobre consumo de frutas e de vegetais de Campbell et al. ${ }^{15}$ Poucas pesquisas têm sido conduzidas na área nutricional relacionadas aos processos de mudança de comportamento. Apenas um estudo especificamente relacionado ao comportamento de comer frutas e hortaliças da população adulta foi encontrado na literatura ${ }^{16}$, e somente um utilizou o modelo para estudar o comportamento alimentar de grupos de adolescentes ao ingerir frutas e hortaliças ${ }^{17}$. Considerando algumas características das frutas e das hortaliças, tais como: alto conteúdo de fibras, antioxidantes e nenhum colesterol ${ }^{18-20}$, e levando em conta que as dietas de adolescentes apresentam baixo conteúdo de fibras e alto conteúdo de gordura saturada ${ }^{21}$ e, além disso, constatando a falta de estudos que venham a investigar esse tipo de comportamento alimentar dos adolescentes, é que se pretende entender o comportamento alimentar de adolescentes na perspectiva do Modelo Transteorético. Especificamente, o objetivo deste estudo foi investigar a relação entre os estágios e processos de mudança propostos por Prochaska, no que se refere ao consumo de frutas e de hortaliças entre adolescentes brasileiros de baixa renda.

\section{MÉ T O D O S}

Este estudo de desenho transversal utilizou uma amostra de 61 adolescentes de um total de 80 participantes potenciais recrutados em um 
programa social, promovido pela parceria da Associação Atlética do Banco do Brasil (AABB) com a comunidade e universidade local, cujo maior objetivo era reintegrar adolescentes nas escolas. Os adolescentes inscritos nesse programa tinham idade entre 10 e 15 anos e apresentavam problemas acadêmicos e comportamentais na escola. Esses indivíduos eram ainda moradores de bairros periféricos, que possuíam baixa renda, e, entre eles, havia casos de alcoolismo, de drogas e de brigas entre os familiares. Este estudo foi aprovado pelo Comitê de Ética da Universidade Federal de Viçosa.

Para a investigação dos estágios e processos de mudança, utilizou-se um questionário baseado no Modelo Transteorético, desenvolvido em estudo similar ${ }^{22}$ o qual apresentava três partes: a primeira categorizava os participantes entre os estágios de mudança para consumir frutas e hortaliças, por meio de algoritmos de estágios de mudança; a segunda parte avaliava os processos de mudança utilizados pelos participantes, por meio de escalas; e a última parte solicitava informações pessoais dos participantes, como idade e ano escolar em que se encontravam. A seguir, fez-se uma descrição detalhada dos instrumentos utilizados.

O algoritmo validado por Oliveira ${ }^{22}$ foi utilizado para caracterizar os estágios de mudança quanto ao consumo de frutas e de vegetais. Após a realização de grupos focais ${ }^{23}$ com adolescentes, a palavra vegetais foi substituída por hortaliças, pois se verificou que essa é a forma pela qual esses alimentos são popularmente conhecidos. 0 algoritmo de estágios de mudança continha alguns itens relacionados ao consumo habitual de frutas e de hortaliças e itens que determinavam a intenção de mudança desse comportamento no período de seis meses ou até mesmo no mês seguinte. A análise do consumo de frutas e de hortaliças foi feita separadamente, somando o número de porções de cada um desses grupos de alimentos consumidos diariamente ${ }^{17}$.

A categorização dos estágios de mudança foi feita caracterizando: em estágio de pré-
- contemplação no consumo de frutas e de hortaliças, os adolescentes que consumiam menos de cinco porções e não pensavam em alterar esse comportamento; em estágio de contemplação, os que tinham intenção de mudança em um período de até seis meses; em estágio de preparação, os que demonstravam a intenção de alterar o comportamento em um mês; e, em estágio de ação ou manutenção, os que consumiam cinco ou mais porções de frutas ou de hortaliças. No último caso, o comportamento de consumir frutas e hortaliças em quantidades adequadas deveria tornar-se um hábito por seis meses ou mais.

A escala usada para medir os processos de mudança usados pelos adolescentes era formada por 10 subescalas, com um total de 40 itens, desenvolvida por Oliveira ${ }^{22}$ em estudo similar. Para efeitos desse estudo, após a realização de cinco grupos focais ${ }^{23}$, verificou-se a necessidade de reduzir o número desses itens para 20. Os indivíduos responderam as questões por meio de uma escala Likert de cinco pontos (nunca, raramente, ocasionalmente, freqüentemente, repetidamente). Essa é uma escala ordinal, que visa medir as atitudes dos adolescentes, cabendo a cada um escolher entre cinco respostas de acordo com as atitudes apresentadas referentes ao consumo de frutas e de hortaliças. As respostas foram pontuadas com valores variando de 1 (nunca) a 5 (repetidamente).

Usando o programa de estatística para as ciências sociais Statistical Program for Social Science (SPSS) ${ }^{24}$ - realizou-se a Análise de Variância (Anova) ${ }^{25,26}$, que é um procedimento para testar a hipótese de que as médias encontradas são iguais, visando investigar diferenças nos usos dos processos de mudança entre adolescentes, nos diferentes estágios de mudança (pré-contemplação/contemplação, preparação e ação/manutenção). Para a análise dos dados, os estágios de mudança foram agrupados em estágios de pré-ação e em estágios finais, sendo o primeiro grupo constituído por pré-contemplação e contemplação (estágios de pré-ação), o segundo por preparação, e o terceiro por ação e manu- 
tenção (estágios finais). O estágio de preparação foi agrupado separadamente dos estágios finais, pois, de acordo com Sorensen et al. ${ }^{1}$, pessoas nesse estágio são mais receptivas às intervenções de educação para a saúde.

\section{RESULTADOS E DISCUSSÃO}

Nas Tabelas 1 e 2, são mostrados os resultados dos efeitos principais dos estágios de mudança, do nível de significância de cada processo de mudança, da média das diferenças entre os estágios de mudança e dos scores dos desvios-padrão dos adolescentes nos estágios de mudança pré-contemplação/contemplação, preparação e ação/manutenção em relação a frutas e a verduras/legumes, respectivamente.

As Análises de Variância (Anova) indicaram que alguns estágios de mudança foram significantemente associados às variáveis dependentes (processos de mudança). Os resultados revelaram diferenças estatísticas significantes $(p<0,05)$ no consumo de frutas, no uso dos processos de mudança autoliberação (indivíduos nos estágios de mudança pré-contemplação/ contemplação e preparação; preparação e ação/ manutenção) e no suporte social (indivíduos nos estágios de mudança pré-contemplação/ contemplação e preparação). Em relação ao consumo de verduras e de legumes, foram encontradas diferenças estatísticas significantes no uso do processo de mudança auto-reavaliação (entre indivíduos nos estágios de mudança pré-contemplação/contemplação e preparação) e no processo autoliberação (indivíduos nos estágios de mudança pré-contemplação/contemplação e preparação, preparação e ação/manutenção). As diferenças encontradas no uso dos processos de mudança no consumo de frutas, de verduras e de legumes evidenciaram que diferentes processos devem ser utilizados em atividades educativas, as quais promovem esses comportamentos alimentares. Por exemplo, os dados apontam que se deve enfatizar o processo suporte social para promover o consumo de frutas entre os indivíduos classificados como pré-contempladores e o processo auto-reavaliação naqueles classificados como contempladores no consumo de vegetais.

Tabela 1. Uso dos processos de mudança de acordo com os estágios de mudança no consumo de frutas.

\begin{tabular}{|c|c|c|c|c|}
\hline \multirow{3}{*}{ Processos de mudança } & \multirow{3}{*}{ F Total $(\mathrm{df}=2,60)$} & \multicolumn{3}{|c|}{ Estágios de mudança } \\
\hline & & $P C / C \quad(n=36)$ & $P(n=13)$ & $\mathrm{A} / \mathrm{M}(\mathrm{n}=12)$ \\
\hline & & $\mathrm{LSM} \pm \mathrm{SEM}$ & $\mathrm{LSM} \pm \mathrm{SEM}$ & $\mathrm{LSM} \pm \mathrm{SEM}$ \\
\hline \multicolumn{5}{|l|}{ Cognitivo/experiencial } \\
\hline Aumento de consciência & 1,59 & $2,4 \pm 1,03$ & $2,8 \pm 1,09$ & $2,9 \pm 1,06$ \\
\hline Alívio dramático & 0,28 & $3,4 \pm 1,17$ & $3,4 \pm 1,25$ & $3,7 \pm 1,45$ \\
\hline Reavaliação ambiental & 1,53 & $3,4 \pm 1,20$ & $3,9 \pm 0,89$ & $3,0 \pm 1,57$ \\
\hline Auto-reavaliação & 1,07 & $3,2 \pm 1,29$ & $3,8 \pm 0,87$ & $3,4 \pm 1,75$ \\
\hline Liberação social & 0,21 & $2,3 \pm 1,12$ & $2,3 \pm 0,96$ & $2,5 \pm 1,18$ \\
\hline \multicolumn{5}{|l|}{ Comportamental } \\
\hline Autoliberação & $5,81 *$ & $2,7 \pm 0,94^{a}$ & $3,4 \pm 1,21^{a, b}$ & $2,0 \pm 0,83^{b}$ \\
\hline Administração de contingência & 0,88 & $3,2 \pm 1,24$ & $3,5 \pm 0,90$ & $3,7 \pm 1,11$ \\
\hline Suporte social & $3,84^{*}$ & $2,8 \pm 1,09^{a}$ & $3,8 \pm 0,75^{a}$ & $3,2 \pm 1,28$ \\
\hline Contracondicionamento & 1,06 & $2,8 \pm 1,27$ & $2,2 \pm 0,75$ & $2,5 \pm 1,17$ \\
\hline Controle de estímulos & 0,85 & $1,8 \pm 1,05$ & $2,0 \pm 0,91$ & $1,4 \pm 1,16$ \\
\hline
\end{tabular}

${ }^{1}$ Anova; $\mathrm{PC} / \mathrm{C}=$ Pré-contemplação/Contemplação; $\mathrm{P}=$ Preparação; $\mathrm{A} / \mathrm{M}=$ Ação/Manutenção; * $p<0,05$.

Mesma letra sobrescrita indica diferença significante, e diferentes letras sobrescritas indicam que não há diferença significante. Least Square Mean (LSM) foi medida em Likert Escala de 5 pontos (1= nunca, 5= repetidamente). 
Tabela 2. Uso dos processos de mudança de acordo com os estágios de mudança no consumo de verduras e legumes.

\begin{tabular}{|c|c|c|c|c|}
\hline \multirow{3}{*}{ Processos de mudança } & \multirow{3}{*}{ F Total $(\mathrm{df}=2,60)$} & \multicolumn{3}{|c|}{ Estágios de mudança } \\
\hline & & $P C / C \quad(n=36)$ & $P(n=13)$ & $\mathrm{A} / \mathrm{M}(\mathrm{n}=12)$ \\
\hline & & $\mathrm{LSM} \pm \mathrm{SEM}$ & LSM \pm SEM & $\mathrm{LSM} \pm \mathrm{SEM}$ \\
\hline \multicolumn{5}{|l|}{ Cognitivo/experiencial } \\
\hline Aumento de consciência & 1,49 & $2,4 \pm 1,08$ & $2,9 \pm 1,10$ & $2,4 \pm 0,72$ \\
\hline Alívio dramático & 1,40 & $3,5 \pm 1,22$ & $3,7 \pm 0,96$ & $2,9 \pm 1,75$ \\
\hline Reavaliação ambiental & 1,96 & $3,5 \pm 1,32$ & $3,6 \pm 1,07$ & $2,6 \pm 1,06$ \\
\hline Auto-reavaliação & $3,06 *$ & $3,0 \pm 1,26^{a}$ & $3,9 \pm 1,08^{a}$ & $3,2 \pm 1,75$ \\
\hline Liberação social & 0,18 & $2,4 \pm 1,15$ & $2,2 \pm 0,98$ & $2,5 \pm 1,22$ \\
\hline \multicolumn{5}{|l|}{ Comportamental } \\
\hline Autoliberação & $3,29 *$ & $2,5 \pm 0,88^{a}$ & $3,2 \pm 1,16^{a, b}$ & $2,2 \pm 1,25^{b}$ \\
\hline Administração de contingência & 1,18 & $3,4 \pm 1,21$ & $3,5 \pm 1,06$ & $2,8 \pm 1,07$ \\
\hline Suporte social & $2,56 *$ & $3,1 \pm 1,11$ & $3,4 \pm 0,92$ & $2,4 \pm 1,41$ \\
\hline Contracondicionamento & 2,36 & $2,8 \pm 1,20$ & $2,5 \pm 1,10$ & $1,9 \pm 0,83$ \\
\hline Controle de estímulos & 2,07 & $1,8 \pm 1,04$ & $2,0 \pm 1,15$ & $1,1 \pm 0,35$ \\
\hline
\end{tabular}

${ }^{1}$ Anova; $\mathrm{PC} / \mathrm{C}=$ Pré-contemplação/Contemplação; $\mathrm{P}=$ Preparação; $\mathrm{A} / \mathrm{M}=\mathrm{Ação/Manutenção;} \mathrm{*} p<0,05$.

Mesma letra sobrescrita indica diferença significante e diferentes letras sobrescritas indicam que não há diferença significante. Least Square Mean (LSM) foi medida em Likert Escala de 5 pontos ( $1=$ nunca, $5=$ repetidamente).

Resultados semelhantes ao deste estudo também foram encontrados por Lamb \& Joshi ${ }^{27}$, que, ao investigarem quatro processos de mudança, encontraram apenas dois deles - $p$ aumento de consciência e a, autoliberação - que apresentaram correlação significativa com todos os estágios. No entanto, os resultados desse estudo se diferem daqueles preconizados por Prochaska $\&$ Velicer ${ }^{10}$, os quais identificaram padrão similar de uso dos processos de mudança entre diferentes comportamentos, como o de fumar e o de ingerir bebidas alcoólicas. Assim, os resultados indicam que os processos de mudança podem ocorrer de maneira diferente nos estágios de mudança, quando se trata de comportamento alimentar. É possível ainda que, em culturas diferentes, alguns processos de mudança sejam mais usados do que em outros ${ }^{28}$. O modelo foi desenvolvido com população americana, ao passo que este estudo foi com adolescentes brasileiros de baixa renda.

De acordo com o preconizado pelo Modelo Transteorético, indivíduos em estágios iniciais de mudança (pré-contemplação e contemplação) usam menos os processos de mudança do que indivíduos em outros estágios. Neste estudo, verificou-se que indivíduos nos estágios de pré-contemplação/contemplação usavam menos os processos autoliberação e auto-reavaliação do que aqueles no estágio de preparação para o consumo de verduras e de legumes.

Quanto ao consumo de frutas, os processos de mudança, autoliberação e suporte social foram menos utilizados por indivíduos em pré-contemplação/contemplação do que por aqueles em preparação. Tais resultados são semelhantes aos encontrados em outros estudos 1 21,29, 30 .

\section{O N CLUS Ã O}

Os dados revelam que adolescentes apresentam particularidades no uso dos processos de mudança, pois alguns dos processos foram utilizados por eles, ao se moverem através dos estágios de mudança, e estavam associados estatisticamente a estes. Com base nesses resultados, sugere-se que sejam realizadas intervenções, visando promover o consumo de frutas e de hortaliças nessa população. Com o intuito de ajudar os indivíduos a se moverem para estágios mais avançados, deve-se estimular o uso dos processos de mudança autoliberação e suporte 
social em relação a consumo de frutas, bem como dos processos auto-reavaliação e autoliberação para o consumo de hortaliças.

Mais estudos devem ser conduzidos, utilizando o Modelo Transteorético no consumo de frutas e de hortaliças em populações de adolescentes brasileiros de diferentes classes sociais, para que os resultados possam ser, então, comparados.

\section{RE FER Ê N CIAS}

1. Soresen G, Stoddart A, Hunt MK, Herbert JR, Ceckene JK, Avrunim JS, et al. The effects of a health promotion - health protection intervention on behavior change. The WellWork study. Am J Public Health. 1998; 88(11):1685-90.

2. Laforge RG, Velicer, WF, Richmonde RL, Owen N. Stage distributions for five health behaviors in the United States and Australia. Prevent Med. 1999; 28(1):61-74.

3. Prochaska JO, DiClemente CC, Norcorss JC. In search of how people change: applications to addictive behaviors. Am Psychol. 1992; 47(9):1102-14.

4. Levesque DA, Miranda D, Kosiak BK, Prochaska JO, Prochaska JM, Cummins CO, et al. [Internet]. Application of the Transtheoretical Model of change to informed choice in the medicare population [cited 27 Sept. 2002]. Available from: www.google.com

5. Hargreaves MK, Schlundt DG, Buchowski MS, Hardy RE, Rossi SR, Rossi JS. Stages of change and the intake of dietary fat in African-American Women: improving stage assignmente using the Eating Style Questionnaire. J Am Diet Assoc. 1999; 99(11):1392-9.

6. Suris AM, Trapp MC, DiClemente CC, Cousing J. Application of the Transtheoretical Model of behavior change for obesity in Mexican American women. Addict Behav. 1998; 23(5):655-68.

7. Lee C. Attitudes, knowledge, and stages of change: a survey of exercise patterns in older Australian women. Health Psychol. 1993; 12(6):476-80.

8. Marcus BH, Simkin LR. The stages of exercise behavior. J Sports Med Physical Fitness. 1993; 33(1):83-9.

9. Marcus BH, Banspach, SW, Lefebvre RC, Rossi JS, Carleton RA, Abrams DB. Using the stages of change model to increase the adoption of physical activity among community participants. Am J Health Promot. 1992; 6(6):624-9.

10. Prochaska JO, Velicer WF. The Transtheoretical Model of health behavior change. Am J Health Promot. 1997; 12(1):38-48.

11. Brug J, Glanz K, Kok G. The relationship between self-efficacy, attitudes, intake compared to others, consumption, and stages of change related to fruit and vegetables. Am J Health Promot. 1997; 12(1):25-30.

12. Steptoe A, Wijetunge S, Doherty S, Wardle J. Stages of change for dietary fat reduction: associations with food intake, decisional balance and motives for food choice. Health Educ J. 1996; 55:108-22.

13. Sporn LA, Contento JR. Stages of change in dietary fat reduction:social psychological correlates. J Nutr Educ. 1995; 27:191-9.

14. Rossi SR, Rossi JS, Rossi-Delprete LM, Prochaska JO, Banspach SW, Carleton RA. Processs of change model for weight control for participant in a comunity - based weight loss programs. Int J Addict. 1994; 29(2):161-77.

15. Campbell MK, DeVellis BM, Strecher VJ, Ammerman AS, De Villes RF, Sandler RS. Improving dietary behavior: the effectiveness of tailored messages in primary care settings. Am J Public Health. 1998; 84(5):783- 7.

16. Chung S, Herr SL. Processes of change differ among stages for eating fruits andvegetables in young adults. Proceedings of the $34^{\text {th }}$ Annual Conference of society for Nutrition Education; 2001. Oakland (CA); 2001.

17. Cullen KW, Bartholomew LK, Parcel GS, Koehly L. Measuring stage of change for fruit and vegetable consumption in 9-to12-year-old girls. J Behav Med. 1998; 21(3):241-54.

18. Campbell MK, Polhamus BMJ, Bennett K, Kalsbeek W, Coole D, Jacson B, et al. Assign fruit and vegetable consumption in a 5 a Day study targeting rural blacks: the issue of portion size. J Am Diet Assoc. 1996; 96:1040-1.

19. Steinmetz KA, Potter JD. Vegetables, fruit, and cancer prevention: a review. J Am Diet Assoc. 1996; 96(10):1027-39.

20. Willet W, Trichopoulus D. Nutrition and cancer: a summary of the evidence. Cancer Causes Control. 1996; 7:178-80.

21. Doyle El, Feldman RHL. Factors affecting nutrition behavior among middle-class adolescents in urban area of Northen region of Brasil. Rev Saúde Pública. 1997; 31(4):342-50.

22. Oliveira MCF. Examining the application of the transtheoretical model of change for fruit and 
vegetable consumption among college students [dissertation]. Fort Collins (CO): Colorado State University; 2002.

23. Frenn M, Malin S, Bansal NK. Stage-based interventions for low fat diet with middle school students. J Pediatr Nurs. 2003; 18(1):36-45.

24. Statistical Package for the Social Science for windows. SPSS/Pc version 10.0. Chicago: SPSS; 2000.

25. Gliner JÁ, Morgan GA. Research methods in applied settings: an integrated approach to design and analysis. Mahwah (NJ): Lawrence Elbaum Associates; 2000.

26. Hinkle DE, Wiersma W, Jurs SG. Applied statistics for the behavioral science. Boston: Houghton Mifflin Company; 1998.

27. Lamb R, Joshi S. The stage model and processes of change in dietary fat reduction. J Hum Nutr Diet. 1996; 9:43-5.
28. Suris AM, Trapp MC, Diclemente CC, Cousin J. Application of the transtheoretical model of behavior change for obesity in Mexican American women. Addict Behav. 1998; 23:655-68.

29. Greene GW, Rossi SR, Reed GR, Willey C, Prochaska JO. Stages of change for dietary fat to $30 \%$ of energy or less. J Am Diet Assoc. 1994; 94(10): 1105-10.

30. Rossi SR, Rossi JS, Prochaska JO. A stage model for reducing dietary fat. In J.O. Prochaska (chair). The Stages of Change: Extensions to New Areas of Behavior Change. Symposium presented at the $98^{\text {th }}$ Annual Conference of the American Psychological Association; Boston; Massachusetts; 1990. Boston (MA): APA; 1990.

Recebido em: 25/10/2004

Versão final reapresentada em: 17/1/2005 Aprovado em: 17/2/2005 